\title{
HER2, estrogen receptor-negative metastatic hidradenocarcinoma: identification of TP53 mutation in both primary and cell-free DNA
}

\author{
Siho Kim ${ }^{1}$, Jinhyun $\mathrm{Cho}^{1}$, Won Park ${ }^{2}$, Sang-Hee Choi ${ }^{5}$, Woongyang Park ${ }^{3}$, \\ Kee-Taek Jang ${ }^{4}$, Jeeyun Lee ${ }^{1}$
}

\author{
${ }^{1}$ Division of Hematology-Oncology, Department of Medicine, Samsung Medical Center, Sungkyunkwan University School of \\ Medicine, Seoul, Korea \\ ${ }^{2}$ Department of Radiation Oncology, Samsung Medical Center, Sungkyunkwan University School of Medicine, Seoul, Korea \\ ${ }^{3}$ Samsung Genome Institute, Seoul, Korea \\ ${ }^{4}$ Department of Pathology and Translational Genomics, Samsung Medical Center, Sungkyunkwan University School of Medicine, \\ Seoul, Korea \\ ${ }^{5}$ Department of Radiology, Samsung Medical Center, Sungkyunkwan University School of Medicine, Seoul, Korea
}

Received: January 19, 2017

Revised: February 24, 2017

Accepted: February 27, 2017

Corresponding author:

Jeeyun Lee

Division of Hematology-

Oncology, Department of

Medicine, Samsung Medical

Center, Sungkyunkwan University

School of Medicine, 81 Irwon-ro,

Gangnam-gu, Seoul 06351, Korea

Tel: +82-2-3410-1779

E-mail:jyunlee@skku.edu

\section{ABSTRACT}

Hidradenocarcinoma is an extremely rare cutaneous skin carcinoma which arise from the sweat gland. There are several report about potential targets in this rare disease including estrogen receptor (ER) and human epidermal growth factor receptor 2 (HER2). A 53-year-old man who presented with a slowly growing mass on the left thumb which was pathologically confirmed of HER2 negative, ER negative hidradenocarcinoma. At the time of presentation, the patient had metastatic lesions to lung and axillary lymph nodes. The patient received several lines of palliative chemotherapy and radiotherapy, but died of the disease progression. We identified TP53 G245S mutation in both primary tumor specimen and cell-free based targeted sequencing. Herein, we report on a very rare case of metastatic hidradenocarcinoma with TP53 G245S mutation but no other treatable targets from clinical sequencing, which pursued an aggressive clinical course.

Keywords: Hidradenocarcinoma; Metastatic disease; P53 mutation
This is an Open Access article distributed under the terms of the Creative Commons Attribution Non-Commercial License (http:// creativecommons.org/licenses/ by-nc/4.0/).

\section{INTRODUCTION}

Hidradenocarcinoma is extremely rare and an aggressive cutaneous skin adnexa carcinoma which arises from the sweat gland. Given the extreme rarity of the disease entity, optimal treatment for hidradenocarcinoma with metastasis has not been defined yet. Based on several reports, hidradenocarcinoma present as clinically painless slowly growing firm cystic nodules which have high metastatic potential to regional lymph nodes, lung, bone, brain, liver, viscera and skin. In 1989, it was reported that hidradenocarcinoma of the scalp was estrogen receptor 
positive and the patient showed nearly complete regression of lymph node metastasis after tamoxifen therapy [1]. In 2007, a 44-year-old man with chest nodule and axillary lymph node metastases demonstrated amplification of human epidermal growth factor receptor 2 (HER2)/neu locus within the tumor and a nodal metastasis [2]. The patient received adjuvant trastuzumab-based chemotherapy and radiotherapy and had complete remission. As of to date, few anecdotal case reports on different regimens for metastatic hidradenocarcinoma have been reported which include paclitaxel, platinum, fluoropyrimidine, doxorubicin, and etoposide. Recently, a whole exome sequencing of a patient with estrogen receptor-positive metastatic hidradenocarcinoma has shown several potentially actionable genomic aberrations such as FGFR1 amplification, $C D H 1$ splice mutation, MYST3 amplification, and ZNF703 mutation [3].

Herein, we report a metastatic hidradenocarcinoma patient who showed negativity for HER2 and estrogen receptor with targeted sequencing profiling to identify potential targets in this rare tumor entity.

\section{METHODS}

The Institutional Review Board of the Samsung Medical Center approved the study. The patient provided informed consent form before study entry. Tumor areas $(>60 \%)$ were dissected under microscopy from $4-\mu \mathrm{m}$-thick unstained sections by comparison with an H\&E stained slide, and genomic DNA was extracted using a Qiagen DNA FFPE Tissue Kit (Qiagen, Hilden, Germany) according to the manufacturer's instructions. After extraction, we measured concentrations and 260/ 280 and 260/230 nm ratios using a spectrophotometer (ND1000, Nanodrop Technologies, ThermoFisher Scientific, Waltham, MA, USA). Tumor tissue DNA was analyzed with targeted sequencing on 381 genes. Illumina HiSeq 2500 (Illumina, San Diego, CA, USA) was used for sequencing with 100 -bp pairedend reads. The sequencing reads were aligned to the human genome reference sequence (hg19) using BWA-mem (v0.7.5), SAMTOOLS (v0.1.18), Picard (v1.93), and GATK (v3.1.1) for sorting SAM/BAM files, duplicate marking, and local realignment, respectively. Local realignment and base recalibration were carried out based on dbSNP137, Mills indels, HapMap, and Omni. SNVs and indels were identified using MuTect (v1.1.4) and Pindel (v0.2.4), respectively. ANNOVAR was used to annotate the detected variants. Only variants with an allele frequency greater than $1 \%$ were included in the results.

For cell-free DNA (cfDNA) sequencing, blood was collected during routine phlebotomy as part of standard cancer care. Blood samples were immediately processed upon receipt to isolate plasma. Plasma was isolated from ethylenediaminetetraacetic acid tubes by centrifugation at $1,600 \times \mathrm{g}$ during 10 minutes at $4^{\circ} \mathrm{C}$. Plasma was aliquoted and stored at $-70^{\circ} \mathrm{C}$. CfDNA was extracted from aliquots $(1 \mathrm{~mL})$ of plasma using the QIAamp circulating nucleic acid kit (Qiagen) with the QIAvac 24 Plus vacuum manifold, following the manufacturer's instructions, and quantified by Qubit fluorometer (Life Technologies, Carlsbad, CA, USA). All cfDNA analysis was performed at Guardant. The Guardant360 Digital Sequencing technology (DST) enables high quality sequencing of each individual circulating DNA fragment in plasma with single molecule sensitivity. The Guardant360 panel sequences 54 target onco-/ tumor-suppressor genes including the complete exonic bases of 18 actionable cancer-related genes and the critical; ("hot") exons of an additional 36 genes (e.g., exons containing at least one or more reported somatic mutations in COSMIC [Catalogue of Somatic Mutations in Cancer] or separate publications), resulting in a concatenated targeted region of approximately 78,000 base pairs (78 kbp). The test simultaneously sequences the 54 cancer-related genes to an average depth of coverage of $8,000 \times[4,5]$.

\section{Clinical sequencing}

Since the patient was diagnosed at disseminated stage and there were only few reports on chemotherapeutic option of the disease, immunohistochemistry $(\mathrm{IHC})$ panel and clinical sequencing were performed. The patient's tumor at diagnosis was sequenced with the targeted 381-gene sequencing panel using formalin-fixed, paraffin-embedded (FFPE). The targeted sequencing demonstrated TP53 G245S mutation. After approximately 1-year of chemotherapy, re-biopsy of the tumor was performed for genomic sequencing but no tumor was identified from the re-biopsied specimen. Thus, cell-free 54-gene panel sequencing using plasma was performed. The cell-free sequencing again demonstrated $7.1 \%$ allele frequency of TP53 G245S. The IHC profile demonstrated negativity for c-met, HER2, estrogen receptor, and tropomyosin receptor kinase A (Fig. 1B-D).

\section{CASE REPORT}

A 53-year-old man with no previous history of illnesses except for diabetes mellitus presented with a slowly growing and asymptomatic mass over a year on the left thumb. The mass was excised and histopathologic diagnosis of the tumor 

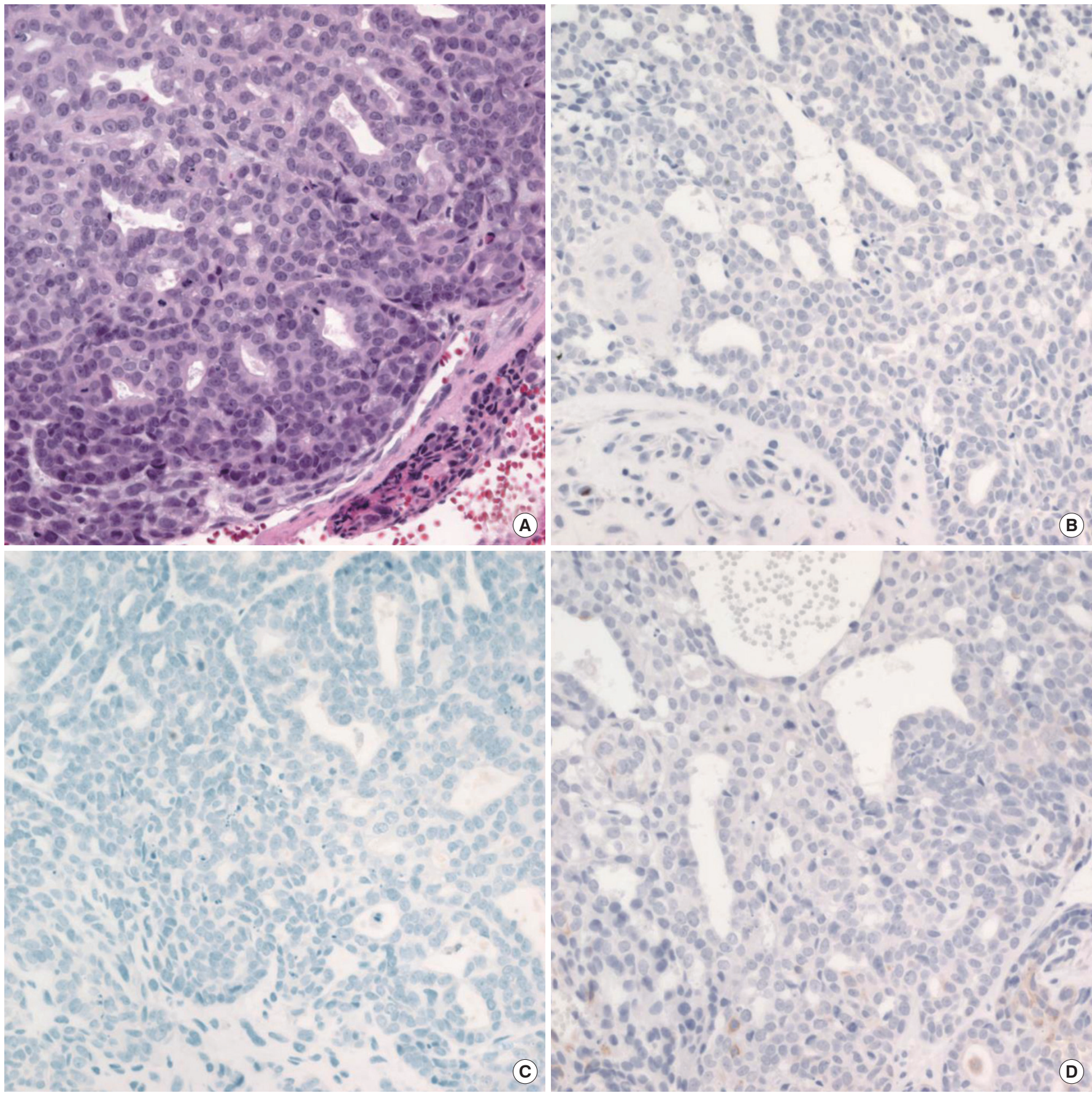

Fig. 1. (A) Histopathologic examination showed sheet-like tumor cell nest and many lumen formations with some decapitation feature, which suggests sweat gland origin. Tumor cells exhibited round nuclei with prominent nucleoli and frequent mitoses. The overall features of histologic findings were compatible with hidradenocarcinoma (H\&E, $\times 400)$. (B) Immunohistochemical study showed negativity for estrogen receptor, (C) human epidermal growth factor receptor 2, and (D) tropomyosin receptor kinase A ( $\times 400)$.

was hidradenocarcinoma (Fig. 1A). At the stage work-up, left upper extremity magnetic resonance imaging and positron emission tomography-computed tomography demonstrated $2.8 \times 4.0 \times 3.6 \mathrm{~cm}$ sized lobulated mass between left 1st proximal phalanx and interphalangeal joint on volar aspect (Fig.
$2 \mathrm{~A})$, multiple heterogeneous size metastatic lymph nodule on left axilla, and at least four discrete nodules on both lung (Fig. 2B). The axillary lymph node was fixed and not movable. Since his disease status was not amenable to curative surgery, the patient received 1st line capecitabine and cisplatin. After four 

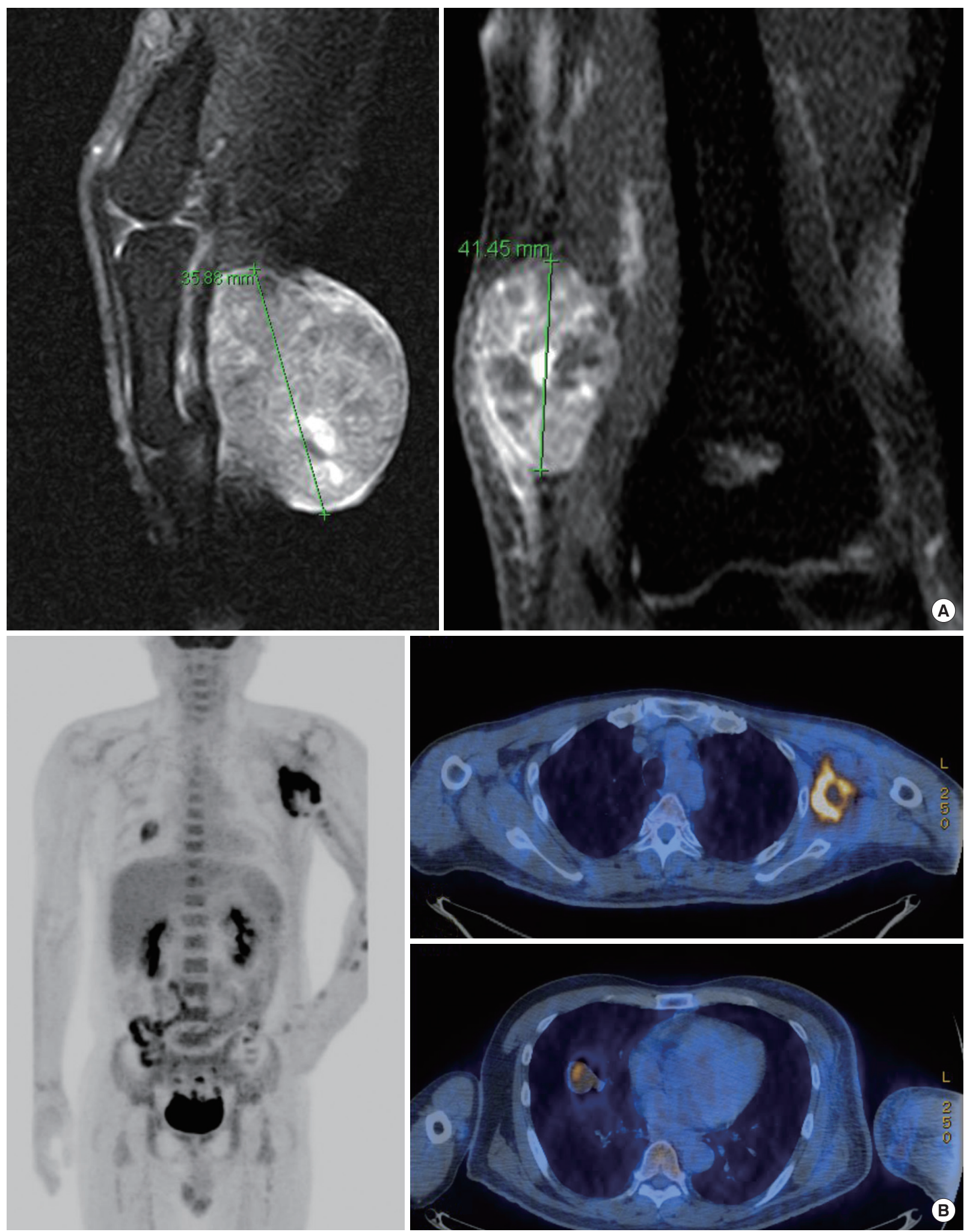

Fig. 2. (A) Left upper extremity magnetic resonance imaging shows $2.8 \times 4.0 \times 3.6 \mathrm{~cm}$ lobulateral mass between left $1 \mathrm{st}$ proximal phalanx and interphalangeal joint on volar aspect and $3 \times 3 \times 4.1 \mathrm{~cm}$ lobulated mass on medial side of left distal humerus. (B) Positron emission tomographycomputed tomography shows multiple heterogeneous size metastatic lymph nodule on left axilla, and at least four discrete nodules on both lung. 
cycles of capecitabine and cisplatin, the metastatic lung nodules were progressive. After two cycles of second-line etoposide and cisplatin, the lung nodules were progressively increasing in size as well as his axillary lymph nodes. He developed symptomatic enlargement of axillary lymph nodes after third-line paclitaxel/cisplatin chemotherapy. The patient received salvage radiotherapy to his axillary lymph nodes, primary mass, and several enlarging extremity masses at 45 to $60 \mathrm{~Gy}$. The axillary masses as well as skin nodules were responsive to radiotherapy with significant reduction in pain and masses. However, after 2 months from completion of radiotherapy, the patient developed progressive disease to lung and kidney with paraneoplastic hypercalcemia. The patient received sunitinib and died of the disease progression 2 months later

\section{DISCUSSION}

Most recently, a systemic literature review was conducted using PubMed, MEDLINE, and PMC, including all articles regarding hidradenocarcinoma up to November 2014 and found that $36 \%, 16 \%, 27 \%, 12 \%$, and $85 \%$ are positive for androgen receptor, progesterone receptor, estrogen receptor, HER2, and epidermal growth factor receptor, respectively [6]. However, in this case, there were no protein expressions identified by IHC.

There is no established optimal chemotherapy for metastatic hidradenocarcinoma probably due to the extreme rarity of the disease. This patient was only modestly responsive to known chemotherapy combination such as etoposide/cisplatin, capecitabine/cisplatin, or paclitaxel/cisplatin. Moreover, the patient did not respond to sunitinib as well. Although there has been an anecdotal case report on two patients with disease stabilization on sunitinib for 8 to 10 months [7], our patient did not respond to sunitinib, however. Because the tumor did not express HER2 or estrogen receptor, HER2-targeted agents [8] or tamoxifen [9], as reported by others, were not considered in this patient.

There was TP53 G245S mutation in our patient. A p53 is mutated in over $50 \%$ of human cancers [10]. They are broadly distributed within the DNA-binding domain (aa 102-292) with six hot spots at codons $175,245,248,249,273$, and 282 , and generate conformationally aberrant proteins with impaired transcriptional function [10]. One study analyzed p53 mutations in 16 sweat gland carcinomas and have identified 5 of 16 contained p53 mutation which showed that p53 protein accumulation was frequent in sweat gland carcinomas (71\%) but rare in sweat gland adenomas. Of the five patients with p53 mutations, there were four missense G:C8A:T transition mutations in two spiradenocarcinomas and two eccrine hidradenocarcinomas, and a $9 \mathrm{bp}$ deletion in a porocarcinoma. Although p53 mutation has been identified, there was no available targeted agent at this time. Of note, the cell-free sequencing of blood matched with tumor specimen sequencing report as described previously [4]. In addition, cell-free sequencing was an extremely useful tool when tumor rebiopsy was failed. Most recently, whole exome sequencing of this tumor revealed protein patched homolog 1 and transcription factor 7 like 1 mutations, which may be potentially actionable [3]. The presence of these mutations should be confirmed in an expanded series of hidradenocarcinoma cases.

Based on our experience, metastatic hidradenocarcinoma was a relatively chemoresistant and aggressive tumor when diagnosed at disseminated stage. There is limited treatment option for this metastatic disease and further sequencing effort should be placed to subdivide into HER2-negative, estrogen receptor-negative hidradenocarcinoma.

\section{CONFLICTS OF INTEREST}

No potential conflict of interest relevant to this article was reported.

\section{ACKNOWLEDGMENTS}

This work was supported by a grant from the Korean Health Technology R\&D Project, Ministry of Health and Welfare, Republic of Korea (HI14C3418).

\section{REFERENCES}

1. Sridhar KS, Benedetto P, Otrakji CL, Charyulu KK. Response of eccrine adenocarcinoma to tamoxifen. Cancer 1989; 64:366-70.

2. Nash JW, Barrett TL, Kies M, Ross MI, Sneige N, Diwan AH, et al. Metastatic hidradenocarcinoma with demonstration of Her-2/neu gene amplification by fluorescence in situ hybridization: potential treatment implications. J Cutan Pathol 2007;34:49-54.

3. Gupta E, Guthrie KJ, Krishna M, Asmann Y, Parker AS, Joseph RW. Whole exome sequencing of a patient with metastatic hidradenocarcinoma and review of the literature. Rare Tumors 2015;7:5719.

4. Kim ST, Lee WS, Lanman RB, Mortimer S, Zill OA, Kim KM, 
et al. Prospective blinded study of somatic mutation detection in cell-free DNA utilizing a targeted 54-gene next generation sequencing panel in metastatic solid tumor patients. Oncotarget 2015;6:40360-9.

5. Lanman RB, Mortimer SA, Zill OA, Sebisanovic D, Lopez R, Blau $S$, et al. Analytical and clinical validation of a digital sequencing panel for quantitative, highly accurate evaluation of cell-free circulating tumor DNA. PLoS One 2015; 10:e0140712.

6. Soni A, Bansal N, Kaushal V, Chauhan AK. Current management approach to hidradenocarcinoma: a comprehensive review of the literature. Ecancermedicalscience 2015;9:517.

7. Battistella M, Mateus C, Lassau N, Chami L, Boukoucha M, Duvillard $\mathrm{P}$, et al. Sunitinib efficacy in the treatment of metastatic skin adnexal carcinomas: report of two pati- ents with hidradenocarcinoma and trichoblastic carcinoma. J Eur Acad Dermatol Venereol 2010;24:199-203.

8. Kazakov DV, Ivan D, Kutzner H, Spagnolo DV, Grossmann P, Vanecek T, et al. Cutaneous hidradenocarcinoma: a clinicopathological, immunohistochemical, and molecular biologic study of 14 cases, including Her2/neu gene expression/amplification, TP53 gene mutation analysis, and $\mathrm{t}(11 ; 19)$ translocation. Am J Dermatopathol 2009;31:23647.

9. Schroder U, Dries V, Klussmann JP, Wittekindt C, Eckel HE. Successful adjuvant tamoxifen therapy for estrogen receptor-positive metastasizing sweat gland adenocarcinoma: need for a clinical trial? Ann Otol Rhinol Laryngol 2004;113:242-4.

10. Hollstein M, Sidransky D, Vogelstein B, Harris CC. p53 mutations in human cancers. Science 1991;253:49-53. 